\title{
Dynamic Contrast-Enhanced MRI of Mouse Liver: A Feasibility Study Using a Dual-Input Two-Compartment Tracer Kinetic Model
}

\author{
Septian Hartono ${ }^{1,2^{*}}$, Choon Hua Thng', Kai Hsiang Chuang ${ }^{3}$, Huynh Hung ${ }^{4}$, Tong San Koh ${ }^{2}$ \\ ${ }^{1}$ School of Electrical \& Electronic Engineering, Nanyang Technological University, Singapore City, Singapore \\ ${ }^{2}$ Department of Oncologic Imaging, National Cancer Centre Singapore, Singapore City, Singapore \\ ${ }^{3}$ Laboratory of Molecular Imaging, Singapore Bioimaging Consortium, Singapore City, Singapore \\ ${ }^{4}$ Laboratory of Molecular Endocrinology, National Cancer Centre Singapore, Singapore City, Singapore \\ Email: ${ }^{*}$ septian.hartono@nccs.com.sg
}

Received 23 December 2014; accepted 6 February 2015; published 13 February 2015

Copyright (C) 2015 by authors and Scientific Research Publishing Inc.

This work is licensed under the Creative Commons Attribution International License (CC BY).

http://creativecommons.org/licenses/by/4.0/

c) (7) Open Access

\begin{abstract}
Dynamic contrast-enhanced magnetic resonance imaging (DCE-MRI) has been widely applied to evaluate microcirculatory parameters in clinical settings. However, pre-clinical studies involving DCE-MRI of small animals remain challenging with the requirement for high spatial and temporal resolution for quantitative tracer kinetic analysis. This study illustrates the feasibility of applying a high temporal resolution ( 2 s) protocol for liver imaging in mice by analyzing the DCE-MRI datasets of mice liver with a dual-input two-compartment tracer kinetic model. Phantom studies were performed to validate the $T_{1}$ estimates derived by the proposed protocol before applying it in mice studies. The DCE-MRI datasets of mice liver were amendable to tracer kinetic analysis using a dual-input two-compartment model. Estimated micro-circulatory parameters were consistent with liver physiology, indicating viability of applying the technique for pre-clinical drug developments.
\end{abstract}

Keywords

DCE-MRI, Mouse, Liver Imaging, Preclinical Imaging

\section{Introduction}

Liver cirrhosis constitutes a final common pathway for a myriad of liver diseases including Hepatitis B, C and

"Corresponding author.

How to cite this paper: Hartono, S., Thng, C.H., Chuang, K.H., Hung, H. and Koh, T.S. (2015) Dynamic Contrast-Enhanced MRI of Mouse Liver: A Feasibility Study Using a Dual-Input Two-Compartment Tracer Kinetic Model. J. Biomedical Science and Engineering, 8, 90-97. http://dx.doi.org/10.4236/jbise.2015.82009 
fatty liver, resulting in significant morbidity and mortality. Although advances in the last 20 years have led to availability of many efficacious drugs that can control Hepatitis B and C, treatment regimens are still suboptimal with high cost, significant side effects and incomplete response. Most treatment guidelines have thus advocated treatment only for those at highest risk of developing cirrhosis while "masterly inactivity" is recommended for those with early liver disease.

The problem in real life scenario however, is the lack of good non-invasive tool that can accurately predict early onset fibrosis and progression to cirrhosis. While non-invasive tests including fibroscan, magnetic resonance elastography and serum based tests have been reported to show fairly good correlation with fibrosis, the sensitivity and specificity of most of these tests are still suboptimal at F2 to F3 fibrosis (metavir score), which unfortunately, is the range upon which clinical decisions are made for treatment [1] [2]. In addition, most of these tests are targeted at the degree of fibrosis, which is a marker of end point damage but does not reflect disease onset or direct pathogenesis.

Accumulating evidence indicates that one of the early pathogenetic processes in liver cirrhosis is capillarisation of liver sinusoidal endothelium or loss of fenestrae. This change is believed to alter the liver sinusoidal dynamics and result in ischemia of hepatocytes and drive the chronic process of injury. By capitalizing on the physical concepts of mono-compartment and dual-compartment kinetics, DCE imaging is able to detect the Space of Disse to indicate whether fibrosis has occurred. Indeed, human studies have shown that patients with normal livers returned a near-zero fractional interstitial space $\left(v_{2}\right)$ due to large fenestrae allowing free exchange of low-molecular weight compounds such as gadolinium-chelate contrast between the vascular space (sinusoids) and interstitial space (Space of Disse) while cirrhotic patients demonstrate a measurable value for the interstitial Space of Disse [3]-[5].

In this work, we explore the feasibility of applying a high spatial and temporal resolution (2 s) DCE-MRI protocol for arterial concentration estimation and perfusion imaging in mice liver. We carried out a pilot study using DCE-MRI to assess the kinetics of normal mouse liver to compute a reliable model that can be subsequently applied in the cirrhotic mice.

\section{Materials and Methods}

\subsection{Mice}

Five male C57BL/6 mice (6 weeks old, $30 \pm 2 \mathrm{~g}$ ) were used in this study. Mouse core body temperature was monitored with a rectal probe and maintained at $37^{\circ} \mathrm{C} \pm 0.3^{\circ} \mathrm{C}$ by a feedback-controlled warm-air heating system. The mice were kept under $1 \%$ - 2\% isoflurane anesthesia during MR scanning.

This study was approved by the local Institutional Animal Care and Use Committee. All mice were maintained according to the guidelines for the Care and Use of Laboratory Animals published by the National Institutes of Health. They were provided with sterilized food and water ad libitum and housed in ventilated cages with air and oxygen.

\subsection{DCE-MRI Protocol}

DCE MRI experiments were performed on a 7-T MR scanner (Bruker ClinScan, Bruker BioSpin MRI GmbH, Ettlingen, Germany) using a three-dimensional (3D) spoiled gradient recalled sequence (FLASH 3D) with the following parameters: repetition time $\mathrm{TR}=3.04 \mathrm{~ms}$, echo time $\mathrm{TE}=1.23 \mathrm{~ms}$, field of view FOV $=36 \times 36 \mathrm{~mm}^{2}$, $128 \times 128$ matrix half-Fourier reconstructed to $256 \times 256,8 \times 1 \mathrm{~mm}^{2}$ slices, final image resolution of $0.14 \times$ $0.14 \times 1 \mathrm{~mm}^{2}$, and acquisition time of $2 \mathrm{~s}$.

Tissue pre-contrast and post-contrast T1 values were estimated using the variable flip angle technique [6] [7]. To estimate the tissue native T1 values, pre-contrast images were acquired with different flip angles: $2^{\circ}, 4^{\circ}, 6^{\circ}$, $8^{\circ}, 10^{\circ}, 12^{\circ}, 14^{\circ}, 16^{\circ}$, and $18^{\circ}$. For each flip angle, 10 repetitions were performed and averaged. The dynamic imaging protocol consists of 10 baseline acquisitions with flip angles $6^{\circ}$ and $14^{\circ}$ (five times each), followed by the manual injection of $50 \mathrm{~mL}$ of gadoterate meglumine, INN (Dotarem, Guerbet S.A., Villepinte, France) through the tail vein and $50 \mathrm{~mL}$ saline flush. One-hundred and thirty post-contrast acquisitions were performed with flip angle $14^{\circ}$ over a period of $260 \mathrm{~s}$, with the contrast injected at the start of the first post-contrast acquisition. 


\subsection{Tracer Kinetic Modeling}

Consider a bi-compartmental tissue system where the first compartment represents the vascular space and the second compartment represents the interstitial space. Assuming well-mixed compartments, tracer concentration in each compartment at time $t$ is given as follows [8]-[10]:

$$
\begin{gathered}
v_{1} \frac{\mathrm{d}}{\mathrm{d} t} C_{1}(t)=F \rho\left(C_{\text {in }}(t)-C_{1}(t)\right)-P S \rho\left(C_{1}(t)-C_{2}(t)\right) \\
v_{2} \frac{\mathrm{d}}{\mathrm{d} t} C_{2}(t)=P S \rho\left(C_{1}(t)-C_{2}(t)\right)
\end{gathered}
$$

where $F$ denotes blood flow, $C_{1}$ and $C_{2}$ denote tracer concentration in the vascular and interstitial space, respectively, PS denotes the permeability surface-area product, and $\rho$ denotes the tissue of density, which is set at 1 $\mathrm{g} / \mathrm{mL} . v_{1}$ is the fractional vascular volume, $v_{2}$ is the fractional interstitial volume, and $C_{\text {in }}(t)$ denotes the arterial input concentration.

The operational equation for analysis of the DCE imaging data can be expressed as

$$
C_{\text {tiss }}(t)=F C_{\text {in }}(t) \otimes R(t)
$$

where $C_{\text {tiss }}$ denotes the tracer concentration in the tissue voxel and $\otimes$ denotes the convolution operator. $R(t)$ is the impulse residue response function, which describes the fractional amount of tracer remaining in the tissue owing due to an impulse input at time $t$. And $R(t)$ is given by

$$
R(t)=A \exp \left(s_{1} t\right)+(1-A) \exp \left(s_{2} t\right)
$$

where $s_{1}$ and $s_{2}$ are the solutions of the following quadratic equation:

$$
S^{2}+\left(\frac{P S \rho}{v_{1}}+\frac{P S \rho}{v_{2}}+\frac{F \rho}{v_{1}}\right) s+\frac{P S \rho}{v_{2}} \times \frac{F \rho}{v_{1}}=0
$$

and

$$
A=\frac{S_{1}+\frac{P S \rho}{v_{1}}+\frac{P S \rho}{v_{2}}}{s_{1}-s_{2}}
$$

\subsection{Dual Input Model}

Consider two sources of blood flow to the liver: flow from the hepatic artery $F_{A}$ and flow from the portal vein $F_{P V}$. Assuming that the tracer concentration-time curves for the hepatic artery $C_{A}(t)$ and the portal vein $C_{P V}(t)$ can be sampled from dynamic MR images, the total input concentration-time curve $C_{i n}(t)$ can be expressed as follows [3]:

$$
C_{\text {in }}(t)=\alpha C_{A}(t)+(1-\alpha) C_{P V}(t)
$$

where $\alpha\left(=F_{A} / F\right)$ denotes the arterial fraction. The total hepatic blood flow, $F$, then is given as the sum of $F_{A}$ and $F_{P V}$.

\subsection{Data Processing}

Post-processing was performed off-line on an Intel ${ }^{\circledR}$ Core $^{\mathrm{TM}} 2$ Duo personal computer with Matlab ${ }^{\mathrm{TM}}$ (MathWorks, Natick, MA). For each case, region of interest corresponding to the liver within the central six (of eight) imaging slices were manually outlined by an experienced radiologist.

The hepatic arterial input $C_{A}(t)$ was approximated by sampling the concentration-time curve at the aorta and the portal venous input $C_{P V}(t)$ was sampled from the portal vein. $C_{A}(t)$ and $C_{P V}(t)$ were divided by a factor of (1 - Hct), where Hct is the fractional hematocrit for mice and was set at 45\% [11] [12].

\section{Results}

A typical set of signal-time concentration profiles in the artery, the portal vein, and the liver tissue was given in 
Figure 1. The profiles were similar with the findings previously reported in human studies [3]. The order of arrival of the contrast was as follow: in the artery, in the portal vein, and in the liver tissue. It showed that the two vascular inputs mix in the sinusoids at different time intervals. However, current temporal resolution of image acquisition of two seconds might not be adequate in delineating the difference clearly.

Microcirculatory parameters derived from the conventional compartmental model and the generalized kinetic model were presented in Table 1 and Table 2.

Extravasation parameters derived from the conventional compartmental model such as $v_{2}$ and PS were zero or close to zero for all cases. They agreed with the murine physiology [13]-[16] and corresponded with the results previously observed in human studies [3]. The liver is a highly vascular organ that consists of a series of fenestrated sinusoids which function as the vascular space. The sinusoids are predominantly supplied by the portal vein (80\%) and supplemented by the hepatic artery (20\%) [17] [18]. They are separated from the liver cords by the Space of Disse, which are comprised of two rows of closely apposed hepatocytes. The Space of Disse may then be considered as an interstitial space within the liver. However, due to the large size of the fenestrae of the sinusoids, low-molecular weight compounds such as gadolinium contrast can trace freely between the vascular space (sinusoids) and the interstitial space (Space of Disse). Both of them then effectively function as a single compartment system [4] [19]. Hence low values of fractional interstitial volume and permeability-surface area product in normal liver were expected, as were observed in this study.

Parametric maps of Mouse 03 were given in Figure 2 and a relatively monotonic distribution of $v_{2}$ and PS could be observed in Figure 2(b) and Figure 2(d), thus confirming the single compartment system of normal liver.

Mean values for vascular perfusion $(F)$ as calculated by the standard two-compartment model ranged from $381.34 \mathrm{~mL} / 100 \mathrm{~mL} / \mathrm{min}$ to $835.54 \mathrm{~mL} / 100 \mathrm{~mL} / \mathrm{min}$. There is over-estimation of the hepatic blood flow as the mean of blood flow for mice liver was previously estimated in other studies at $180 \mathrm{~mL} / 100 \mathrm{~mL} / \mathrm{min}$ [20]. The mean values for fractional blood volume $\left(v_{1}\right)$ as calculated by the standard two-compartment model ranged from $5.91 \%$ to $16.38 \%$. There is under-estimation of the values of the percentage of mice hepatic blood volume, as this was previously estimated at $23 \%$ - $36 \%$ [21].

Mean values for $\alpha$ derived by the conventional compartmental model ranged from $43.76 \%$ to $66.80 \%$. There is over-estimation of $\alpha$ as Davies and Morris [18] reported arterial fraction of $19.44 \%$, while Brown reported arterial fraction of $28.77 \%$ [21].

The mono-compartmental system can be inferred as well from the signal concentration-time curve profile in the liver tissue in Figure 3, as a contrast uptake enhancement pattern of a rapid increase which was followed by

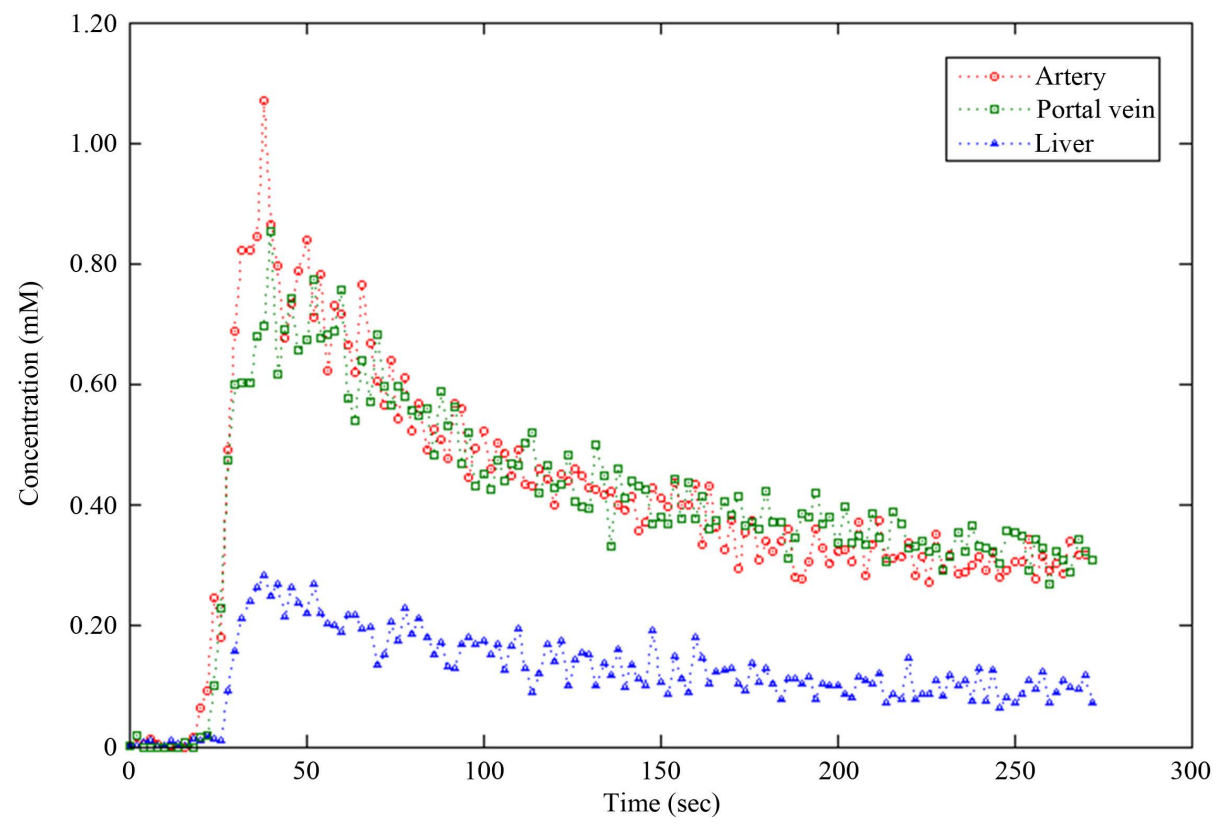

Figure 1. Signal concentration time-curves in the artery, portal vein, and liver tissue of Mouse 01. 
Table 1. Median values of microcirculatory parameters estimated by the standard twocompartmental model (mean values \pm standard deviations, with median values in parentheses).

\begin{tabular}{ccccccc}
\hline \multicolumn{2}{c}{ 2-compartment model } & M01 & M02 & M03 & M04 & M05 \\
\hline$F$ & $(\mathrm{~mL} / 100 \mathrm{~mL} / \mathrm{min})$ & 512.65 & 682.41 & 285.52 & 326.31 & 674.17 \\
$v_{1}$ & $(\%)$ & 13.91 & 14.62 & 6.25 & 8.04 & 15.53 \\
$v_{2}$ & $(\%)$ & 0.01 & 0.01 & 0.00 & 0.35 & 0.00 \\
$P S$ & $(\mathrm{~mL} / 100 \mathrm{~mL} / \mathrm{min})$ & 0.29 & 0.26 & 0.00 & 3.31 & 0.09 \\
\hline
\end{tabular}

Table 2. Median values of microcirculatory parameters estimated by the generalized kinetic model (mean values \pm standard deviations, with median values in parentheses).

\begin{tabular}{ccccccc}
\hline \multicolumn{2}{c}{ Generalized kinetic model } & M01 & M02 & M03 & M04 & M05 \\
\hline$K_{\text {trans }}$ & $(\mathrm{mL} / 100 \mathrm{~mL} / \mathrm{min})$ & 5.71 & 9.07 & 3.02 & 3.31 & 10.32 \\
$v_{\mathrm{e}}$ & $(\%)$ & 13.96 & 13.92 & 7.11 & 9.34 & 14.57 \\
\hline
\end{tabular}

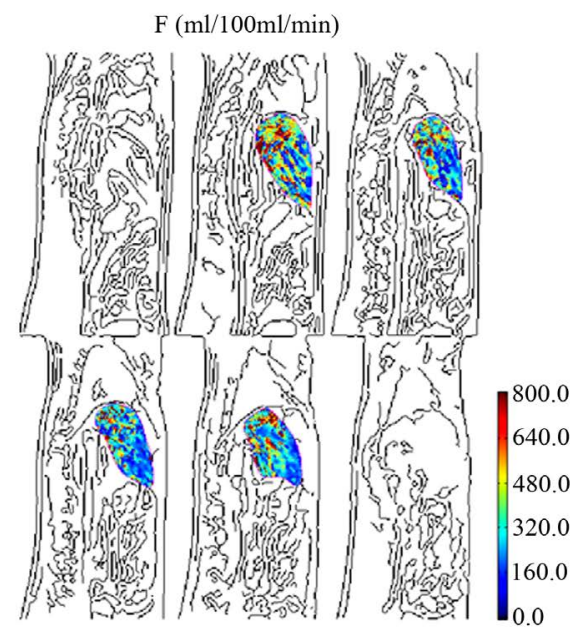

(a)

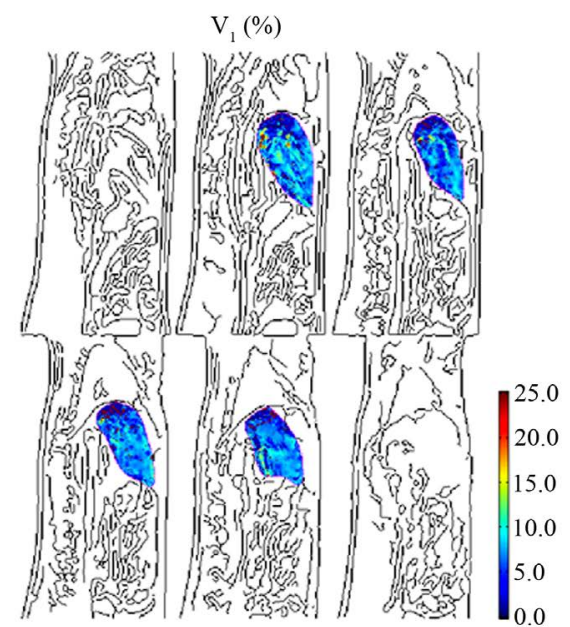

(c)

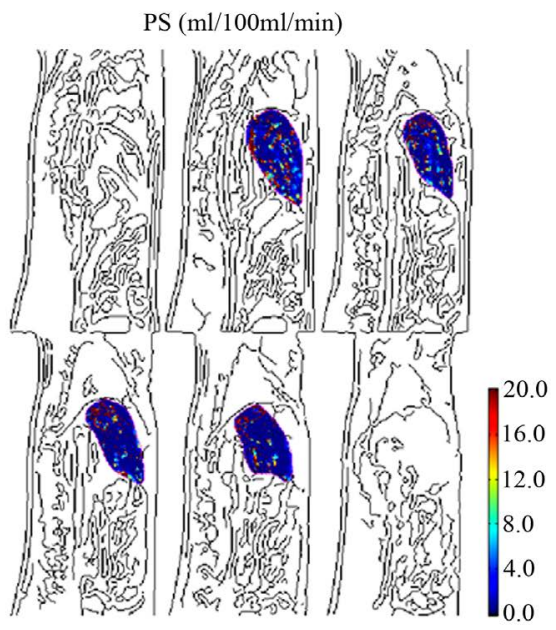

(b)

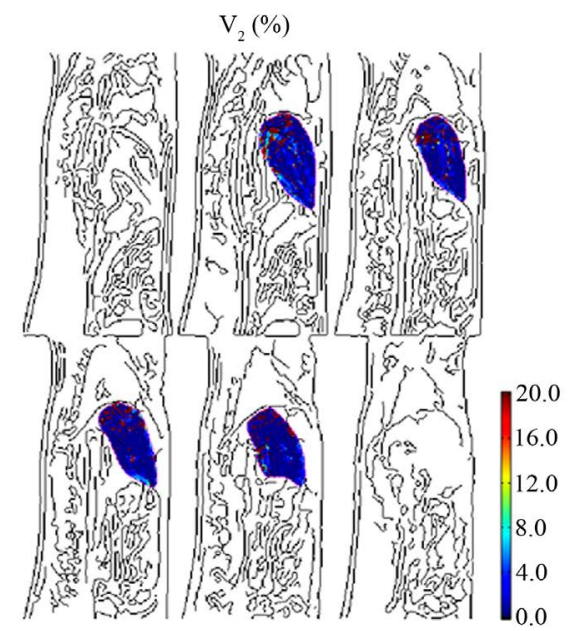

(d)

Figure 2. Parametric maps of (a) blood flow, (b) permeability-surface area product, (c) fractional intravascular space, and (d) fractional extravascular space for Mouse 03 (slice 2 to 7). 


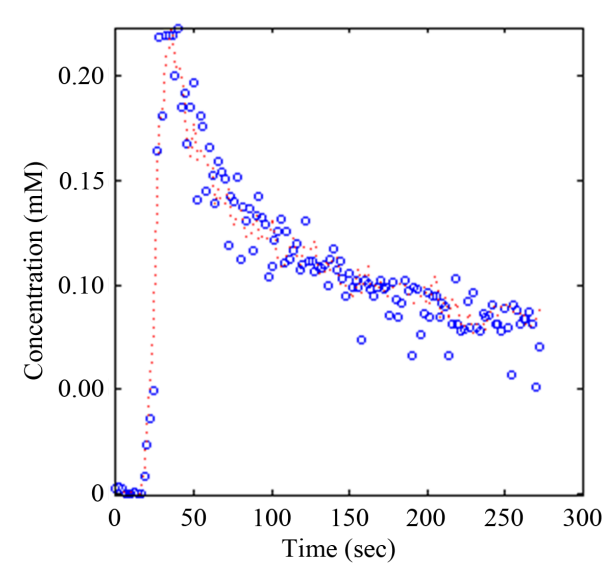

(a)

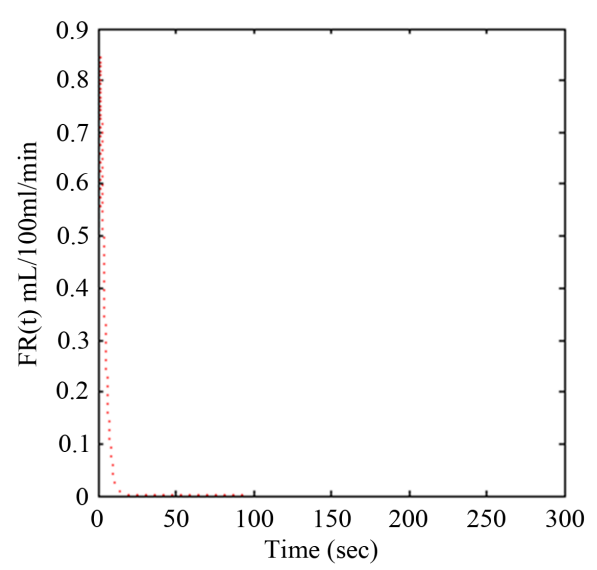

(b)

\begin{abstract}
Figure 3. (a) Example of fitting the dual-input standard two-compartmental model to liver tissue concentration-time curve and (b) impulse residual function $R(t)$ corresponding to the fitting of the enhancement pattern. $F=220.6 \mathrm{~mL} / 100 \mathrm{~mL} / \mathrm{min}, t_{1}=2.58 \mathrm{sec}, \alpha=25.7 \%, v_{1}=9.5 \%, v_{2}=0 \%$, $P S=0$.
\end{abstract}

rapid washout was observed [22]. Figure 3 also showed an example of fitting the dual-input conventional compartmental model to liver tissue concentration-time curve and the impulse residual function $R(t)$ corresponding to the fitting of the enhancement pattern.

\title{
4. Discussion
}

In this study we observed the low values of interstitial space and permeability in all mice, which are concordant with the normal liver physiology.

The shape of the signal concentration-time curve in the liver tissue is also consistent with a single-compartment system, as is shown in Figure 3(a). A contrast uptake enhancement pattern of a rapid increase was followed by rapid washout [22]. Figure 3(b) also showed an example of fitting the dual-input two-compartment model to liver tissue concentration-time curve and the impulse residual function $R(t)$ that is the result of the fitting of the enhancement pattern. It showed an impulse residual function which immediately dropped to zero after the vascular phase, consistent with the behavior of a single-compartment system.

Compared to previously published values of mice hepatic microcirculation, the current model over-estimates hepatic blood flow and under-estimates percentage of mice hepatic blood volume. An over-estimation of arterial fraction, $\alpha$, was also observed.

The over-estimation of $\alpha$ might be due to difficulty in finding a voxel which corresponds to the portal vein for all dynamic images and hence a worse signal-to-noise ratio from the portal vein, which possibly increased the contribution from the arterial input.

To illustrate this problem, the conventional compartmental model was applied as well to analyze the parameters in muscle of Mouse 04 to show the difference of the tracer kinetic behaviors and microcirculatory parameters values in liver and muscle. The values of the microcirculatory parameters were compared in Table 3 . The value of blood flow in muscle was significantly less than in liver. The values of v2 and PS were not zero or close to zero. Davies and Morris [18] reported that the blood flow in muscle was estimated at $91 \mathrm{~mL} / 100 \mathrm{~mL} / \mathrm{min}$. It was similar to what was observed in Mouse 04 at $112.07 \pm 71.01 \mathrm{~mL} / 100 \mathrm{~mL} / \mathrm{min}$. Brown [21] reported that the percentage of vascular space in muscle was estimated at $3 \%-5 \%$. It also agrees with what is observed in Mouse 04 at $5.68 \% \pm 4.14 \%$. The relatively smaller error in the estimation of $\mathrm{F}$ and $\mathrm{v} 1$ in muscle perhaps indicated a higher technical difficulty in applying tracer kinetic modeling in liver since it incorporated not one input signal as is in the muscle but two input signals, aorta and portal vein, for data fitting, which might also be exacerbated by the similar shape of arterial and venous input curves, as was shown in Figure 1.

Although there are technical challenges to this technique, the low values of the mice interstitial space are concordant with observations in humans and lend potential for the use of this quantitative technique as a potential biomarker for sinusoidal capillarization and fibrosis in the Space of Disse. Currently, ultrasound and MR 
Table 3. Microcirculatory parameters in liver and muscle for Mouse 04 (mean values \pm standard deviations, with median values in parentheses).

\begin{tabular}{cccc}
\hline & Mouse 04 & Liver & Muscle \\
\hline$F$ & $(\mathrm{~mL} / 100 \mathrm{~mL} / \mathrm{min})$ & $381.34 \pm 234.06(326.31)$ & $112.07 \pm 71.01(93.83)$ \\
$t_{1}$ & $(\mathrm{~s})$ & $1.98 \pm 2.26(1.47)$ & $5.26 \pm 7.09(2.86)$ \\
$v_{1}$ & $(\%)$ & $8.03 \pm 4.42(8.04)$ & $5.68 \pm 4.14(4.71)$ \\
$v_{2}$ & $(\%)$ & $2.71 \pm 9.75(0.35)$ & $9.29 \pm 4.74(8.85)$ \\
$P S$ & $(\mathrm{~mL} / 100 \mathrm{~mL} / \mathrm{min})$ & $13.26 \pm 22.79(3.31)$ & $14.11 \pm 10.18(13.00)$ \\
\hline
\end{tabular}

elastography have been used as non-invasive techniques for the assessment of fibrosis and cirrhosis. Although advanced fibrosis and cirrhosis can be accurately determined with both modalities, challenges remain for the assessment of early fibrosis.

Our current findings set the stage for future studies with thioacetamide induced liver fibrosis and cirrhosis to determine if DCE-MRI can serve as an imaging biomarker for this important process.

\section{References}

[1] Huwart, L., Peeters, F., Sinkus, R., Annet, L., Salameh, N., terBeek, L.C., Horsmans, Y. and Van Beers, B.E. (2006) Liver Fibrosis: Non-Invasive Assessment with MR Elastography. NMR in Biomedicine, 19, 173-179. http://dx.doi.org/10.1002/nbm.1030

[2] Huwart, L., Sempoux, C., Salameh, N., Jamart, J., Annet, L., Sinkus, R., Peeters, F., terBeek, L.C., Horsmans, Y. and Van Beers, B.E. (2007) Liver Fibrosis: Noninvasive Assessment with MR Elastography Versus Aspartate Aminotransferase-to-Platelet Ratio Index. Radiology, 245, 458-466. http://dx.doi.org/10.1148/radiol.2452061673

[3] Koh, T.S., Thng, C.H., Lee, P.S., Hartono, S., Rumpel, H., Goh, B.C. and Bisdas, S. (2008) Hepatic Metastases: In Vivo Assessment of Perfusion Parameters at Dynamic Contrast-Enhanced MR Imaging with Dual-Input Two-Compartment Tracer Kinetic Model. Radiology, 249, 307-320. http://dx.doi.org/10.1148/radiol.2483071958

[4] Thng, C.H., Koh, T.S., Collins, D.J. and Koh, D.M. (2010) Perfusion Magnetic Resonance Imaging of the Liver. World Journal of Gastroenterology, 16, 1598-1609. http://dx.doi.org/10.3748/wjg.v16.i13.1598

[5] Koh, T.S., Thng, C.H., Hartono, S., Lee, P.S., Choo, S.P., Poon, D.Y.H., Toh, H.C. and Bisdas, S. (2009) Dynamic Contrast-Enhanced CT Imaging of Hepatocellular Carcinoma in Cirrhosis: Feasibility of a Prolonged Dual-Phase Imaging Protocol with Tracer Kinetics Modeling. European Radiology, 19, 1184-1196. http://dx.doi.org/10.1007/s00330-008-1252-y

[6] Wang, H.Z., Riederer, S.J. and Lee, J.N. (1987) Optimizing the Precision in T1 Relaxation Estimation Using Limited Flip Angles. Magnetic Resonance in Medicine, 5, 399-416. http://dx.doi.org/10.1002/mrm.1910050502

[7] Brookes, J.A., Redpath, T.W., Gilbert, F.J., Needham, G. and Murray, A.D. (1996) Measurement of Spin-Lattice Relaxation Times with FLASH for Dynamic MRI of the Breast. The British Journal of Radiology, 69, 206-214. http://dx.doi.org/10.1259/0007-1285-69-819-206

[8] Hayton, P., Brady, M., Tarassenko, L. and Moore, N. (1997) Analysis of Dynamic MR Breast Images Using a Model of Contrast Enhancement. Medical Image Analysis, 1, 207-224. http://dx.doi.org/10.1016/S1361-8415(97)85011-6

[9] Brix, G., Bahner, M.L., Hoffmann, U., Horvath, A. and Schreiber, W. (1999) Regional Blood Flow, Capillary Permeability, and Compartmental Volumes: Measurement with Dynamic CT-Initial Experience. Radiology, 210, 269-276. http://dx.doi.org/10.1148/radiology.210.1.r99ja46269

[10] Port, R.E., Knopp, M.V., Hoffmann, U., Milker-Zabel, S. and Brix, G. (1999) Multicompartment Analysis of Gadolinium Chelate Kinetics: Blood-Tissue Exchange in Mammary Tumors as Monitored by Dynamic MR Imaging. Journal of Magnetic Resonance Imaging, 10, 233-241. http://dx.doi.org/10.1002/(SICI)1522-2586(199909)10:3<233::AID-JMRI3>3.0.CO;2-M

[11] McDonald, T., Clift, R. and Cottrell, M. (1992) Large, Chronic Doses of Erythropoietin Cause Thrombocytopenia in Mice. Blood, 80, 352-358.

[12] Trune, D.R., Kempton, J.B. and Gross, N.D. (2006) Mineralocorticoid Receptor Mediates Glucocorticoid Treatment Effects in the Autoimmune Mouse Ear. Hearing Research, 212, 22-32. http://dx.doi.org/10.1016/j.heares.2005.10.006

[13] Wisse, E. (1970) An Electron Microscopic Study of the Fenestrated Endothelial Lining of Rat Liver Sinusoids. Journal of Ultrastructure Research, 31, 125-150. http://dx.doi.org/10.1016/S0022-5320(70)90150-4 
[14] Wisse, E. (1972) An Ultrastructural Characterization of the Endothelial Cell in the Rat Liver Sinusoid under Normal and Various Experimental Conditions, as a Contribution to the Distinction between Endothelial and Kupffer Cells. Journal of Ultrastructure Research, 38, 528-562. http://dx.doi.org/10.1016/0022-5320(72)90089-5

[15] Smedsrød, B., De Bleser, P.J., Braet, F., Lovisetti, P., Vanderkerken, K., Wisse, E. and Geerts, A. (1994) Cell Biology of Liver Endothelial and Kupffer Cells. Gut, 35, 1509-1516. http://dx.doi.org/10.1136/gut.35.11.1509

[16] Baratta, J., Ngo, A., Lopez, B., Kasabwalla, N., Longmuir, K. and Robertson, R. (2009) Cellular Organization of Normal Mouse Liver: A Histological, Quantitative Immunocytochemical, and Fine Structural Analysis. Histochemistry and Cell Biology, 131, 713-726. http://dx.doi.org/10.1007/s00418-009-0577-1

[17] Chiandussi, L., Greco, F., Sardi, G., Vaccarino, A., Ferraris, C. and Curti, B. (1968) Estimation of Hepatic Arterial and Portal Venous Blood Flow by Direct Catheterization of the Vena Porta through the Umbilical Cord in Man: Preliminary Results. Acta Hepatosplenologica, 15, 166-171.

[18] Davies, B. and Morris, T. (1993) Physiological Parameters in Laboratory Animals and Humans. Pharmaceutical Research, 10, 1093-1095. http://dx.doi.org/10.1023/A:1018943613122

[19] Pandharipande, P.V., Krinsky, G.A., Rusinek, H. and Lee, V.S. (2005) Perfusion Imaging of the Liver: Current Challenges and Future Goals. Radiology, 234, 661-673. http://dx.doi.org/10.1148/radiol.2343031362

[20] Larson, K.B., Markham, J. and Raichle, M.E. (1987) Trace-Kinetic Models for Measuring Cerebral Blood Flow Using Externally Detected Radiotracers. Journal of Cerebral Blood Flow \& Metabolism, 7, 443-463. http://dx.doi.org/10.1038/jcbfm.1987.88

[21] Brown, R.P., Delp, M.D., Lindstedt, S.L., Rhomberg, L.R. and Beliles, R.P. (1997) Physiological Parameter Values for Physiologically Based Pharmacokinetic Models. Toxicology and Industrial Health, 13, 407-484. http://dx.doi.org/10.1177/074823379701300401

[22] Koh, T.S., Thng, C.H., Hartono, S., Kwek, J.W., Khoo, J.B.K., Miyazaki, K., Collins, D.J., Orton, M.R., Leach, M.O., Lewington, V. and Koh, D.M. (2011) Dynamic Contrast-Enhanced MRI of Neuroendocrine Hepatic Metastases: A Feasibility Study Using a Dual-Input Two-Compartment Model. Magnetic Resonance in Medicine, 65, $250-260$. http://dx.doi.org/10.1002/mrm.22596 
Scientific Research Publishing (SCIRP) is one of the largest Open Access journal publishers. It is currently publishing more than 200 open access, online, peer-reviewed journals covering a wide range of academic disciplines. SCIRP serves the worldwide academic communities and contributes to the progress and application of science with its publication.

Other selected journals from SCIRP are listed as below. Submit your manuscript to us via either submit@scirp.org or Online Submission Portal.
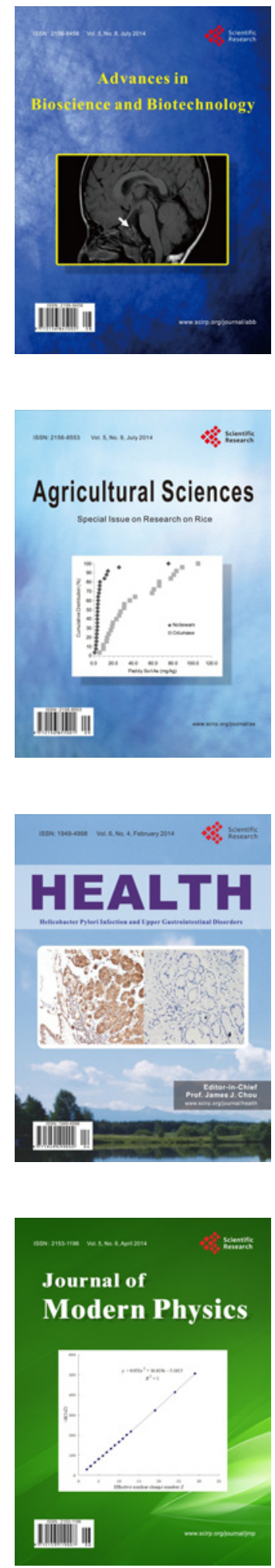
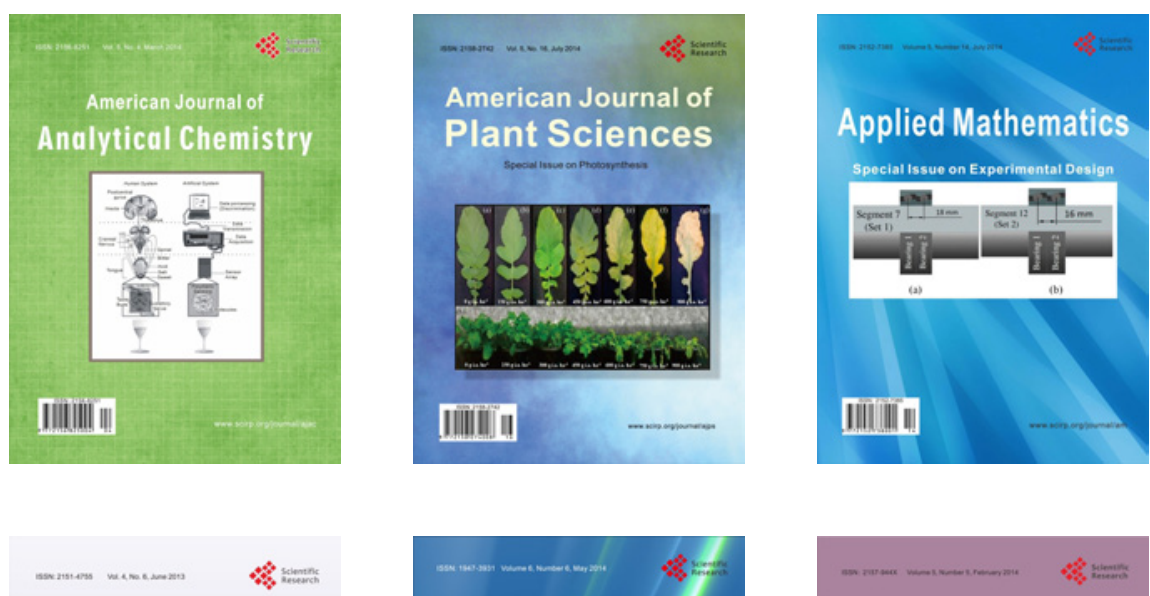

Creative Education
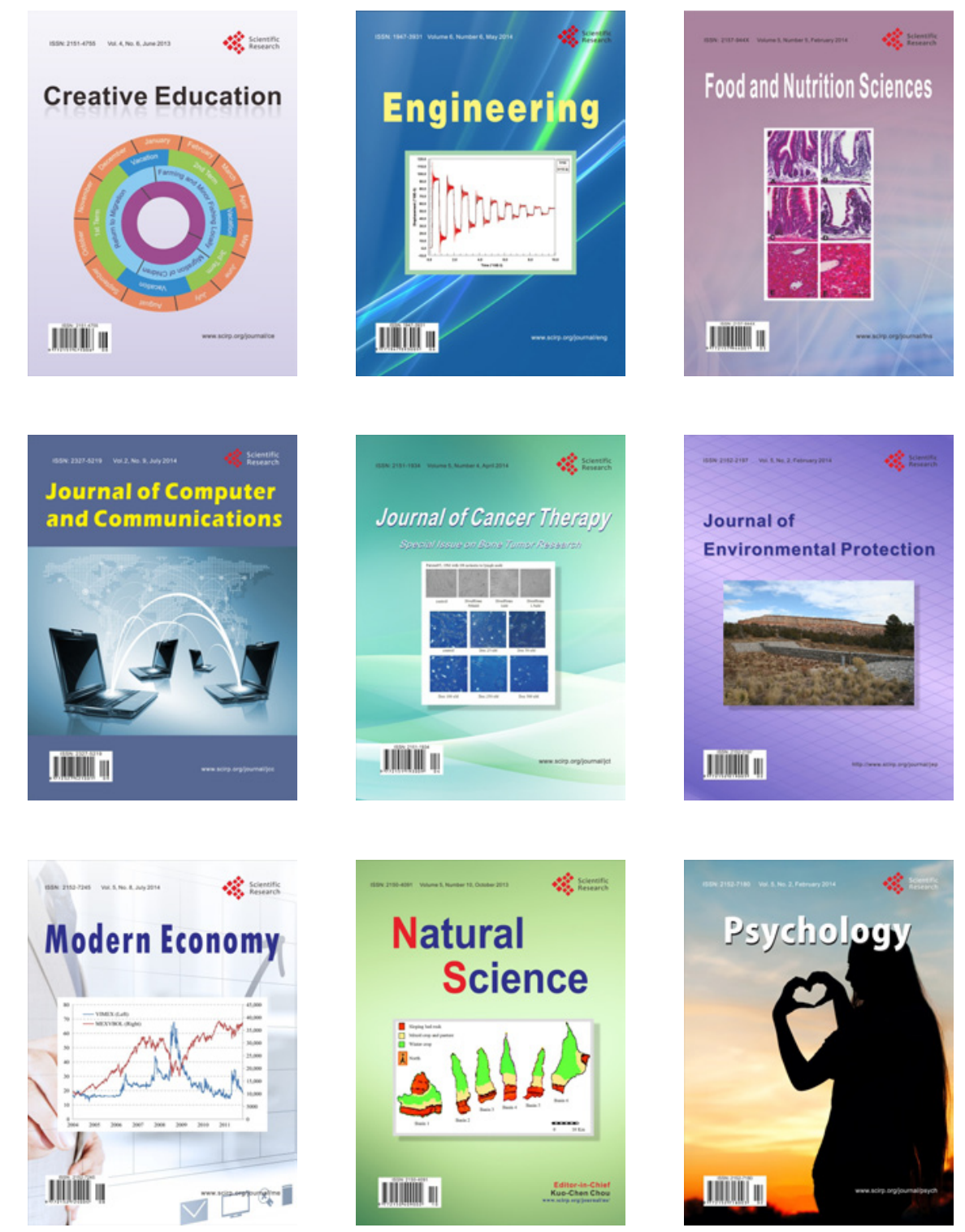